Ann. Sci. For., 1985, 42 (4), 411-434

\title{
Étude complémentaire sur l'influence de la fertilisation sur la qualité du bois de pin maritime (Expérience de Mimizan)
}

\author{
H. POLGE \\ avec la collaboration technique de Thérèse Hurpenu, A. Perrin et P. Gelhay: \\ Station de Recherches sur la Qualité des Bois \\ Centre de Recherches forestières de Nancy, Champenoux, F 54280 Seichanps
}

\begin{abstract}
Résumé
Sur 105 arbres répartis en 5 blocs, 7 traitements de fertilisation el 3 catégories de grosseur, ont été découpées 2 barrettes de $7,4 \times 7,4 \mathrm{~mm}$, une côté bois de compression (Est) et une côté opposé, subdivisées en échantillons coeur et écorce; pour la densité du bois, la séparation des traitements avec et sans phosphore n'est pas nette; les petits arbres donnent le bois le moins dense, et le côté Est le plus dense; il existe des différences significatives entre traitements pour l'épaisseur des barrettes débitées avec des scies-fraises jumelées; les retraits radial et tangentiel sont plus faibles côté cour que côté écorce, et plus forts à l'Ouest qu'à l'Est, l'inverse étant vrai pour le retrait axial ; les engrais agissent sur les retraits, mais la séparation des éléments fertilisants n'est pas claire.

Sur éprouvettes normalisées, les traitements se séparent assez bien, notamment pour la contrainte de rupture en flexion statique et la dureté, où les modalités avec phosphore ont une résistance inféricure.

Une analyse densitométrique laite sur des barrettes de $1,6 \mathrm{~mm}$ d'épaisseur montre une disparition plus précoce à l'Est qu'à l'Ouest de l'effet favorable du phosphore sur la largeur des cernes; l'influence de la grosseur des arbres sur cette largeur augmente avec l'âge; lanisotropie des accroissements est plus marquée dans les traitements avec $P$ seul ou combiné ; il existe des différences significatives globales entre traitements pour les densités moyennes maximales et minimales annuelles, sans regroupement en fonction des éléments apportés; cependant, certaines années, l'ensemble des modalités avec $P$ se sépare de l'ensemble sans P, toujours par des valeurs plus faibles. L'anisotropie des largeurs de cernes augmente dans le temps, mais celle des densités moyonnes annuelles diminue.

Au total, l'influence de la fertilisation sur la qualité des bois, qui n'a jamais été dramatique, a pratiquement disparu dans les derniers cernes produits sur des arbres de 21 ans.
\end{abstract}

\section{Introduction}

L'expérience de fertilisation de Mimizan (département des Landes) a été entreprise sur des plants issus de semis effectués au printemps 1957 ; elle comporte 7 traitements (témoins et apports d'engrais N, P, NK, NP, PK et NPK) et 5 répétitions (blocs complets équilibrés). 
Elle a déjà fait l'objet de nombreux comptes rendus :

- Une première et très importante étude (Guinaudeau et al., 1963) décrivait en détail le dispositif et en analysait les premiers résultats dans des domaines très variés : croissance en hauteur après 2 et 6 saisons de végétation, couverture vivante. survie au terme de la première année d'expérience, production de cônes mâles et femelles, durée de vie et composition chimique des aiguilles, mycorhization...

- Un bilan dendrométrique portant à la fois sur les croissances en hauteur et en circonférence, ainsi que sur la production en volume depuis l'origine a par la suite été établi par Gelpe \& Guinaudenu (1974).

En ce qui concerne plus particulièrement la qualité du bois, plusieurs études successives ont été entreprises, à différents stades de la vie du peuplement. pour voir en particulier si une dégradation trop importante des caractéristiques technologiques ne risquait pas de venir contrebalancer, au moins en partie, les gains de croissance spectaculaires apportés par la fertilisation phosphatée :

- Une première analyse a été faite à l'âge de 7 ans sur des caroties de sondage prélcvées à $40 \mathrm{~cm}$ de hauteur dans les témoins et dans les placeaux ayant bénéficié seulement d'un apport de phosphore (Polge, 1969); la fertilisation entrainait alor's une perte de densité du bois de $6 \mathrm{~g} / \mathrm{dm}^{3}$ par rapport à un échantillonnage systématique de témoins et de $23 \mathrm{~g} / \mathrm{dm}^{3}$ lorsque l'on se référait à des témoins de diamètre comparable, toutes les différences étant significatives; les deux traitements ne se distinguaient pas l'un de l'autre par les densités minimales ou maximales annuelles, mais par le pourcentage de largeur de cerne de densité supéricure à $500 \mathrm{~g} / \mathrm{dm}^{3}$ qui tombait de 30 p. 100 chez les témoins à 24 p. 100 dans les placeaux à phosphore.

- A 9 ans (même document), d'autres analyses ont été faites sur les arbres enlevés à l'occasion de la première éclaircie, en travaillant, d'une part sur les premiers arbres abattus dans chaque ligne (échantillonnage systématique), el d'autre part sur les 5 plus gros arbres coupés dans chaque parcelle unitaire (échantillonnage se rapprochant au point de vue dimensions des arbres restant après éclaircie); les 7 traitements se différenciaient mieux les uns des autres au point de vue densité du bois dans le premier cas que dans le second, et le faisaient également davantage sur les 3 derniers cernes que sur la totalité des accroissements; en général les traitements sans phosphore donnaient du bois de densité supérieure, les écarts entre les extrêmes variant de 21 à $34 \mathrm{~g} / \mathrm{dm}^{3}$; il n'apparaissait pas de différences significatives de longueurs de libres, mais un rendement en fibres accru des placeaux $P$ par rapport aux témoins.

- Une autre étude a été entreprise dans ce même dispositif à l'âge de 12 ans et à l'âge de 15 ans (ОhrA et al., 1983); dans le premier cas (matériel constitué par un billon par traitement et par bloc provenant d'un arbre de diamètre voisin de la moyenne de la parcelle unitaire correspondante), il n'y avait aucun effet du traitement sur l'hétérogénéité du bois, ni sur le retrait tangentiel, mais une influence significative sur les retraits radial et volumétrique, sans cependant que soient regroupés de façon nette les traitements avec phosphore d'une part, et sans phosphore d'autre part ; à 15 ans, l'expérience a porté sur 5 billons par parcelle unitaire, représentant bien la variabilité des diamètres du peuplement de chacune d'elles : l'excentricité de la moelle était plus marquée dans les placeaux à phosphore que sans phosphore, mais, inversement, la conicité des tiges y était plus faible; la densité du bois et les divers critères de résistance mécanique des parcelles NP et NPK étaient inféricurs 
à ceux des parcelles $N$, NK et des témoins, mais les traitements $P$ et PK venaient s'intercaler parmi ces dernières.

- Enfin, dans un dernier compte rendu (OHrA et al., 1985) les mêmes auteurs montrent que le phosphore amène une augmentation du rendement en fibre dans le bois de compression, mais une diminution dans le bois normal, et également que, dans le traitement NPK par exemple, si le bois de compression a un rendement par unité de poids sec de bois plus faible que le bois opposé, ceci est compensé par une densité plus élevée, en sorte que le rendement par unité de volume est pratiquement inchangé.

Le but de la petite étude complémentaire dont il est rendu compte ici est de vérifier à un âge plus avancé la réalité de quelques observations relativement surprenantes faites précédemment et de suivre l'évolution dans le temps de certaines propriétés du bois de signification technologique majeure.

\section{Matériel et méthodes}

Cette nouvelle expérience a été entreprise sur du matériel tiré d'arbres abattus au printemps 1978 (donc âgés de 21 ans); l'échantillonnage a porté sur 3 individus par bloc et par traitement, à savoir un arbre ayant la circonférence moyenne de chaque parcelle unitaire et deux autres ayant la circonférence moyenne plus ou moins une fois l'écart-type, soit un total de 105 individus, à la partie inférieure desquels ont été prélevés des billons de 65 à $70 \mathrm{~cm}$ de longueur, en excluant la zone trop proche du collet où le fil du bois est fortement dévié.

De rondelles débitées à la partie supérieure des billons, ont été extraites à laide de scies-fraises jumelées, deux séries de barrettes diamétrales suivant la direction de l'excentricité maximale, qui ont par suite fourni côté grand rayon des échantillons de bois de compression, et côté opposé, des échantillons de bois normal. La première série, sciée à des épaisseurs voisines de $7,4 \mathrm{~mm}$ dans les directions tangentielle et axiale, a servi à des mesures de rétractibilité et d'infra-densité, en séparant sur chaque éprouvette radiale les 11 derniers cernes qui ont fourni les échantillons référencés "écorce» et l'ensemble des autres accroissements (échantillons « cour») ; sur la totalité des 420 échantillons ( 7 traitements $\times 5$ blocs $\times 3$ individus $\times 2$ rayons $\times 2$ positions) ont été mesurées à l'aide d'un capteur micrométrique atutomatisé (FErrand, 1981) les dimensions tangentielles et axiales à l'état sec à l'air (après conditionnement à 12 p. 100 d'humidité dans une étuve hygrométrique), puis à l'état saturé, dont ont été tirés les retraits correspondants ; on a procédé de même pour le retrait radial à partir de mesures de longueurs faites avee un comparateur avec une précision du $\mathrm{mm} / 100$; les infra-densités ont été déterminées sur la base du poids anhydre et du poids maximum obtenu après saturation intégrale par le vide.

L'autre série de barrettes a été sciée à une épaisseur de $1,6 \mathrm{~mm}$ dans le sens axial et a servi, après radiographie, à évaluer par analyse densitométrique (Polcr:, 1966) les largeurs de cernes, les densités moyennes, maximales et minimales annuelles, ainsi que l'écart-type des densités à l'intérieur des cernes qui caractérise l'hétérogénéité du bois, ceci pour les 13 accroissements de 1965 à 1977 inclusivement.

Par ailleurs ont été débitées dans les billons des éprouvettes normalisées pour essais mécaniques, pour la localisation desquelles il a été impossible de suivre un 
schéma précis, en raison de la présence fréquente de nœuds ou autres défauts de structure; cn règle générale, on a obtenu 6 éprouvettes par billon : doux du côté «bois de compression », une du côté opposé et 3 en direction perpendiculaire, mais de nombreuses exceptions se sont produites, ne permettant pas de prendre en comptc le facteur "position » dans l'analyse des résultats; les éprouvettes ont d'abord été soumises à des essais en flexion statique suivant la norme NF B $51-008$ at terme desquels ont été mesurées les flèches et les charges à la rupture, à des essais en compression axiale (NF B51-007) et de dureté (NF B51-013) sur des éprouvettes de $6 \mathrm{~cm}$ de longueur débitées dans les parties intactes des éprouvettes précédemment testées en flexion statique, enfin à des déterminations de densités suivant NF B51-005.

\section{Résultats}

\subsection{Barrettes de $7.4 \times 7.4 \mathrm{~mm}$}

Les résultats ont fait l'objet d'analyses de variance faisant la part de 4 sources principales de variation : traitement, taille des arbres (circonférence moyenne $\mathrm{C}=$ moyens, $\overline{\mathrm{C}}+{ }_{\sigma} \mathrm{C}=$ gros, $\overline{\mathrm{C}}-{ }_{\pi} \mathrm{C}=$ petits), côté (tendu et comprimé) et position (cour et écoree), ainsi que des différentes interactions; celles-ci étant. dans tous les cas, rares, faibles (bien que parfois significatives) et difficilement interprétables, il n'en sera pas fait état ci-dessous.

\subsection{Infra-densité}

Elle apparaît comme soumise à l'influence du traitement $\left(\mathrm{F}=7,47^{* *}\right)$, de la taille $\left(F=10,93^{* *}\right)$, du côté $\left(\mathrm{F}=67,91^{* *}\right)$ et de la position $\left(\mathrm{F}=173,87^{* * *}\right)$.

La comparaison des moyennes des traitements par le test de Bonferonı donne les résultats suivants :

$\begin{array}{lccccccc}\text { NP } & P & \text { N } & \text { NPK } & \text { NK } & \text { T } & \text { PK } & \\ 375 & 378 & 391 & 393 & 395 & 397 & 409 & \left(\mathrm{en} g / \mathrm{dm}^{3}\right)\end{array}$

(dans un but de simplification, on se limite ici, et on se limitera par la suite dans les comparaisons de moyennes aux différences significatives au scuil de 5 p. 100).

Contrairement aux premières expériences, les traitements avec et sans phosphore ne se séparent plus de façon nette; on retrouve, comme dans l'étude de OHTA, KELLER \& JANIN (1983), des intercalations d'autant moins explicables qu'elles concernent des traitements différents, et que la hiérarchie des diverses modalités de fertilisation n'est pas la même; on peut maintenant dire que l'effet dépressif du phosphore sur la densité du bois est peu marqué, et observer une certaine tendance à une influence positive du potassium, puisque dans les trois couples NP-NPK, P-PK et $N-N K$, le traitement avec $K$ fournit des valeurs d'infra-densité supérieures.

L'influence de la taille se manifeste comme suit :

\begin{tabular}{ccc} 
Petits & Gros & Moyens \\
381 & 395 & 397 \\
\hline
\end{tabular}


La plus faible densité des petits arbres, qui surprend à première vue, pourrait s'expliquer par le fait qu'après une période juvénile où leur croissance aurait été assez semblable à celle des autres catégories, ils auraient formé des cernes plus étroits à partir du moment où ils auraient subi leur concurrence, de sorte que. sur des barrettes parallélépipédiques, le volume de bois adulte plus dense serait relativement plus réduit.

En ce qui concerne l'effet "côté », on observe sans surprise une densité plus élevée dans le bois de compression $\left(404 \mathrm{~g} / \mathrm{dm}^{3}\right)$ que dans le bois opposé $\left(378 \mathrm{~g} / \mathrm{dm}^{3}\right)$.

De même, comme on pouvait s'y attendre, on trouve une densité beaucoup plus forte dans les échantillons "écorce» $\left(412 \mathrm{~g} / \mathrm{dm}^{3}\right)$ que dans les échantillons «cœur » $\left(370 \mathrm{~g} / \mathrm{dm}^{3}\right)$.

\subsection{Dimensions tangentielles et axiales des éprouvettes}

On pourrait croire qu'en débitant les échantillons à l'aide de scies-fraises jumelées (donc à écartement constant), on obtienne des éprouvettes de même épaisseur, aux erreurs de mesure près; il n'en est rien, et l'on trouve ici, en direction tangentielle, des effets significatifs du traitement $(F=11,93 * *)$, du côté ( $F=5,33 *$ ) et de la position $\left(F=3,82^{*}\right)$, et, en direction axiale, un effet du traitement $\left(F=4,23^{* * *}\right)$.

La comparaison des moyennes des diverses modalités de fertilisation donne les résultats suivants :

Direction tangentielle

$\begin{array}{cccccccc}\mathrm{T} & \mathrm{NP} & \mathrm{P} & \mathrm{NK} & \mathrm{N} & \mathrm{NPK} & \mathrm{PK} & \\ 7374 & 7380 & 7395 & 7398 & 7413 & 7415 & 7421 & \text { microns }\end{array}$

Direction axialc

\begin{tabular}{cccccccc}
$\mathrm{T}$ & $\mathrm{NP}$ & $\mathrm{NK}$ & $\mathrm{P}$ & $\mathrm{N}$ & $\mathrm{PK}$ & NPK & \\
7356 & $\mathbf{7 3 7 4}$ & 7384 & 7384 & 7384 & 7394 & 7398 & microns \\
\hline
\end{tabular}

On observe d'abord que, quel que soit le traitement, les dimensions tangentielles sont supérieures aux dimensions axiales; ceci pourrait être dû (ARCHER, communication personnelle) à la valeur inférieure à 1 du coefficient de Poisson liant une déformation dans le sens du fil du bois à la déformation résultante dans la direction des couches daccroissement, ou encore (KAUMAN, communication personnclle) à l'interaction outil-fibres, l'action d'une dent de fraise ne s'exerçant pas de façon identique dans le sens transversal (où elle coupe les trachéides perpendiculairement à leur axe) et dans le sens longitudinal (où elle peut, soit les sectionner parallèlement à celui-ci, soit les ćcarter les unes des autres à la manière d'un coin).

Par ailleurs, les dimensions axiales et tangentielles sont liées par une corrélation très étroite ( $r=0,90^{* * *}$ ), ce qui laisse à penser que les déformations par rapport à l'épaisseur théorique correspondant à l'écartement des scies-fraises, qui se produisent dans les deux directions considérées, sont occasionnées par une même causc, la structure du bois; on aurait dès Jors un phénomène analogue à celui dont il a déjàt été rendu compte (Polgri, 1978), dans lequel les déformations d'une dent-rabot lors de l'enlèvement d'un copeau étaient fortement corrélées à la densité maximale annuelle 
des cernes successifs $\left(r=0,65^{* *}\right.$ à $\left.0,75^{* * *}\right)$; ici, on ne dispose que d'une seule donnée, relativement grossière, pour caractériser la structure : l'infradensité; elle ne donne pas de liaisons significatives avec les dimensions des éprouvettes, mais une tendance apparaît pour une augmentation de celles-ci avec la densité $(r=0,50$ avec l'épaisseur dans le sens tangenticl qui est, des deux considérées, lá plus variable).

Les différences observées quant aux effets des deux autres sources de variation sur les dimensions tangentielles vont également dans le même sens, puisque l'on a toujours une épaisseur plus forte quand la densité est elle-même plus élevée : 7404 microns pour le bois de compression contre 7395 pour le côté opposé, el 7403 microns pour les échantillons «écorce" contre 7395 pour les échantillons « coeur».

\subsection{Retrail tangentiel}

11 est soumis à un effet traitement $(F=4,68 *$ ), à un effet côté ( $F=23.5 \%$ ) et à un effet position ( $\mathrm{F}=376,8^{*}:$.

L'influence du traitement de fertilisation se présente comme suit :

\begin{tabular}{ccccccccc} 
NP & N & P & NK & NPK & T & PK & & \\
3,40 & 3,52 & 3,70 & 3,74 & 3,79 & 3,82 & 3,86 & $($ en & $\%)$ \\
\hline
\end{tabular}

Pas plus que les autres variables étudiées, le retrait tangentiel, qui est une caractéristique physique très importante pour la plupart des utilisations, ne permet de séparer les traitements avec et sans phosphore; comme il en est généralement, il suit assez bien les variations de l'infradensité $(r=0,75)$, et paraît également aggravé par le potassium, au vu des comparaisons NP-NPK, N-NK et P-PK.

Le retraik tangentiel est plus faible dans le bois de compression que du côté opposé (3,54 p. 100 contre 3,83 p. 100), en accord avec la littérature (Low, 1964), el également moins élevé dans l'échantillon cœur que dans l'échantillon écorce (3,10 p. 100 contre 4,27 p. 100$)$, en conformité avec le gradient positif généralement observé chez les résineux du bois juvénile au bois adulte.

\subsection{Retrait radial}

Il apparaît comme dépendant à la fois du traitement $(F=57,23 * *)$, de la taille $(F=12,37 *)$, du côté $(F=98,19 \% *)$ et de la position $(F=12,12 \%)$.

Les traitements se séparent en 4 groupes ne se recoupant pas, mais l'interprétation des résultats n'en est pas plus facile pour autant, puisque, par exemple, le témoin occupe une position médiane et que les 4 traitements avec phosphore se répartissent par groupes de deux dans les plus forts et les plus faibles retraits.

La comparaison des tailles d'arbres se présente comme suit :

$\begin{array}{cccc}\text { Gros } & \text { Moyens } & \text { Petits } & \\ 2,36 & 2,52 & 2,62 & (\text { en } \%)\end{array}$


Comme le retrait tangentiel, le retrait radial est plus élevé du côté opposé que du côté comprimé $(2,70$ p. 100 contre 2,31 p. 100$)$ et vers l'écorce $(2,57$ p. 100) que vers le cœur $(2,44$ p. 100$)$, avec les mêmes explications probables.

\subsection{Retrait axial}

11 est soumis à l'influence de toutes les sources de variation étudiées : traitement $\left(F=3,25^{* *}\right)$, taille $(F=5,48 * *)$, coté $\left(F=56,33^{* *}\right)$ et position $\left(F=6,16^{*}\right)$.

La comparaison des moyennes de traitement donne :

$\begin{array}{cccccccc}\mathrm{T} & \mathrm{NK} & \mathrm{P} & \mathrm{NPK} & \mathrm{NP} & \mathrm{N} & \mathrm{PK} & \\ 0,11 & 0,12 & 0,15 & 0,18 & 0,19 & 0,19 & 0,28 & \text { (en \%) }\end{array}$

On n'a toujours pas d'effet très net des éléments fertilisants ; on peut seulement observer que, contrairement à une tendance assez générale suivant laquelle les retraits en direction du fil du bois et perpendiculairement à celui-ci varient en sens opposé, ici le traitement PK apparaît comme conférant au bois une forte instabilité dimensionnelle, aussi bien axiale que radiale ou tangentielle.

L'influence de la taille des arbres est donnée par la comparaison :

\begin{tabular}{ccc} 
Petits & Gros & Moyens \\
0,12 & 0,19 & 0,21 \\
\hline
\end{tabular}

On a ici un résultat opposé à celui trouvé pour le retrait radial, puisque ce sont les petits qui se distinguent des deux autres catégories, au lieu des gros.

Le retrait axial est plus de trois fois plus élevé du côté «bois de compression » $(0,26$ p. 100$)$ que du côté opposé $(0,08 \mathrm{p} .100)$, et ceci confirme bien que le bois formé en direction du plus grand rayon dans le pin maritime (en général à l'Est) est effectivement du bois de réaction (Polge \& ILLY, 1967).

Le retrait dans le sens du fil du bois est plus faible dans la zone «écorce» que dans la zone «cœur», c'est-à-dire que, comme pour la source de variation précédente, il suit un gradient de signe contraire aux retraits radial et tangentiel.

\subsection{Barrettes de $1,6 \mathrm{~mm}$ d'épaisseur}

Le but de la partic de l'expérience les concernant était de suivre l'évolution des caractéristiques de structure du bois au cours des 13 années convenablement représentées sur l'ensemble des échantillons.

\subsection{Largeur des accroissements annuels}

Le tableau 1 indique après l'année la valeur du test « $F$ » correspondant, puis les résultats du lest de comparaison des moyennes au seuil de probabilité de 5 p. 100.

On retrouve, ce que laissaient présager les résultats des expériences successives, une séparation de moins en moins nette des traitements avec et sans phosphore : les 
6 premières années, à l'exception de 1967 , les deux groupes se séparent complètement l'un de l'autre, puis, même si la croissance des placeaux avec phosphore demeure la plus rapide, on observe des cas d'absence de différence significalive entre traitements ayant ou non bénéficié de l'apport de cet élément fertilisant, enfin se produisent en 1976 et 1977 des inversions, avec, en particulier, des accroissements très faibles dans la modalité à phosphore seul.

TABLEAU 1

Test de comparaison des moyennes de largeurs de cernes (valeurs exprimées en $\mathrm{mm} / 50$ ).

Multiple comparisons test of ringwidth (values given in $\mathrm{mm} / 50$ ).

\begin{tabular}{|c|c|c|c|c|c|c|c|c|}
\hline 1965 & $\left(16,0^{* *}\right)$ & $\begin{array}{c}P \\
268\end{array}$ & $\begin{array}{l}N P \\
252\end{array}$ & $\begin{array}{l}\mathrm{PK} \\
250\end{array}$ & $\begin{array}{c}N{ }^{3} K \\
249\end{array}$ & $\begin{array}{c}T \\
201\end{array}$ & $\begin{array}{l}\text { NK } \\
178\end{array}$ & $\begin{array}{c}N \\
172\end{array}$ \\
\hline 1966 & $(10,7 * *)$ & $\begin{array}{c}P \\
241\end{array}$ & $\begin{array}{c}\text { Ni'K } \\
234\end{array}$ & $\begin{array}{l}\mathrm{NP} \\
230\end{array}$ & $\begin{array}{l}\mathrm{FK} \\
227\end{array}$ & $\begin{array}{c}T \\
184 \\
-\end{array}$ & $\begin{array}{c}N \\
177 \\
\end{array}$ & $\begin{array}{l}\text { NK } \\
158\end{array}$ \\
\hline 1967 & $(4,4 * *)$ & $\begin{array}{l}\text { PK } \\
225\end{array}$ & $\begin{array}{c}P \\
223\end{array}$ & $\begin{array}{c}\text { NPi } \\
21:\end{array}$ & $\begin{array}{l}N P \\
209 \\
\end{array}$ & $\begin{array}{l}N \\
198\end{array}$ & $\begin{array}{c}\Gamma \\
175\end{array}$ & $\begin{array}{l}\text { NK } \\
166\end{array}$ \\
\hline 1968 & $\left(12,6^{* * *}\right)$ & $\begin{array}{l}\text { PK } \\
207\end{array}$ & $\begin{array}{c}P \\
202 \\
\end{array}$ & $\begin{array}{l}N P \\
201\end{array}$ & $\begin{array}{c}\text { NPK } \\
200\end{array}$ & $\begin{array}{c}\mathrm{N} \\
154 \\
\end{array}$ & $\begin{array}{c}\mathrm{T} \\
1.36\end{array}$ & $\begin{array}{l}\text { NK } \\
133 \\
\end{array}$ \\
\hline 1969 & $\left(8,5^{* * * ;}\right)$ & $\begin{array}{l}\text { PK } \\
194\end{array}$ & $\begin{array}{c}P \\
192\end{array}$ & $\begin{array}{c}\text { NFK } \\
184\end{array}$ & $\begin{array}{l}\text { NP } \\
183\end{array}$ & $\begin{array}{c}N \\
139\end{array}$ & $\begin{array}{l}\text { NK } \\
138\end{array}$ & $\begin{array}{c}\mathbf{T} \\
130\end{array}$ \\
\hline 1970 & $\left(12,1^{* * *}\right)$ & $\begin{array}{c}P \\
197 \\
\end{array}$ & $\begin{array}{l}\text { NP } \\
194\end{array}$ & $\begin{array}{l}\text { PK } \\
193\end{array}$ & $\begin{array}{c}\text { NFK } \\
188\end{array}$ & $\begin{array}{l}\text { NK } \\
147 \\
\end{array}$ & $\begin{array}{c}N \\
144\end{array}$ & $\begin{array}{c}\mathrm{T} \\
136 \\
\end{array}$ \\
\hline 1971 & $\left(12,6^{* *}\right)$ & $\begin{array}{l}\text { NP } \\
156\end{array}$ & $\begin{array}{c}\text { NPK } \\
149\end{array}$ & $\begin{array}{l}\text { PK } \\
142\end{array}$ & $\begin{array}{c}P \\
139\end{array}$ & $\begin{array}{c}\mathrm{T} \\
116 \\
- \\
\end{array}$ & $\begin{array}{c}N \\
110\end{array}$ & $\begin{array}{l}\text { NK } \\
106\end{array}$ \\
\hline 1972 & $\left(3,2^{* * *}\right)$ & $\begin{array}{l}N P \\
132\end{array}$ & $\begin{array}{c}\text { NPK } \\
123 \\
\end{array}$ & $\begin{array}{l}\mathrm{F} \mathbf{K} \\
117\end{array}$ & $\begin{array}{c}\mathrm{P} \\
111\end{array}$ & $\begin{array}{c}\mathrm{T} \\
110 \\
\end{array}$ & $\begin{array}{l}N \\
104\end{array}$ & $\begin{array}{l}\text { NK } \\
102\end{array}$ \\
\hline 1973 & $\left(4,6^{* * *}\right)$ & $\begin{array}{l}\text { NP } \\
135\end{array}$ & $\begin{array}{l}\text { NPK } \\
125\end{array}$ & $\begin{array}{c}P \\
121\end{array}$ & $\begin{array}{l}\text { PK } \\
120\end{array}$ & $\begin{array}{c}\mathrm{N} \\
105\end{array}$ & $\begin{array}{c}T \\
103\end{array}$ & $\begin{array}{l}\text { NK } \\
102\end{array}$ \\
\hline 1974 & $(2,4 *)$ & $\begin{array}{l}\text { NP } \\
156\end{array}$ & $\begin{array}{c}\text { NPK } \\
143\end{array}$ & $\begin{array}{c}P \\
136\end{array}$ & $\begin{array}{l}\text { FK } \\
133\end{array}$ & $\begin{array}{c}T \\
130\end{array}$ & $\begin{array}{l}N \\
1.27\end{array}$ & $\begin{array}{l}N_{K} \\
125\end{array}$ \\
\hline 1975 & $(1,7 \mathrm{NS})$ & $\begin{array}{l}\text { NP } \\
145\end{array}$ & $\begin{array}{c}\text { NPK } \\
134\end{array}$ & $\begin{array}{c}\Gamma \\
120\end{array}$ & $\begin{array}{l}\mathrm{FK} \\
117\end{array}$ & $\begin{array}{c}N \\
116\end{array}$ & $\begin{array}{c}P \\
115\end{array}$ & $\begin{array}{l}\text { NK } \\
100\end{array}$ \\
\hline 1977 & $\left(2,9^{* * *}\right)$ & $\begin{array}{c}\text { NPK } \\
95\end{array}$ & $\begin{array}{r}\mathrm{NP} \\
94\end{array}$ & $\begin{array}{l}N \\
91\end{array}$ & $\begin{array}{l}\top \bar{\Gamma} \\
8,4\end{array}$ & $\begin{array}{r}\text { PK } \\
79\end{array}$ & $\begin{array}{l}P \\
74\end{array}$ & $\begin{array}{r}\text { NK } \\
67\end{array}$ \\
\hline
\end{tabular}


Les valeurs du test «F » de l'effet «côté » pour les différentes années sont les suivantes :

$\begin{array}{cccccccccccccc}1965 & 1966 & 1967 & 1968 & 1969 & 1970 & 1971 & 1972 & 1973 & 1974 & 1975 & 1976 & 1977 \\ 29^{* *} & 54^{* * *} & 61^{* *} & 98^{* *} & 99^{* *} & 174^{* *} & 149^{* *} & 148^{* *} & 192^{* * *} & 184^{* *} & 147^{* *} & 193^{* * *} & 86^{* *}\end{array}$

Il apparaît assez nettement que l'anisotropie, c'est-à-dire la différence de vitesse de croissance sur deux rayons opposés, a, contrairement à ce que l'on aurait pu penser, tendance à augmenter avec l'âge.

11 en est de même pour l'influence de la taille des arbres :

$\begin{array}{ccccccccccccc}1965 & 1966 & 1967 & 1968 & 1969 & 1970 & 1971 & 1972 & 1973 & 1974 & 1975 & 1976 & 1977 \\ 13^{* *} & 12^{* *} & 9^{* *} & 16^{* *} & 14^{* *} & 31^{* *} & 25^{* *} & 33^{* *} & 36^{* * *} & 57^{* *} & 39^{* *} & 54^{* *} & 36^{* * *}\end{array}$

mais, dans ce cas, on retrouve une notion bien connue des sylviculteurs, à savoir que les dominants deviennent de plus en plus dominants, et, les dominés, de plus en plus dominés.

La figure 1 représente les variations de largeurs des cernes en fonction de l'âge pour le bois de compression (côté Est) et pour le bois opposé (côté Ouest), d'une part pour les 4 traitements avec phosphore, d'autre part pour les 3 traitements sans phosphore, avec les droites de régression correspondantes : on voit que l'effet de cet élément a cessé d'être significatif dès 1971 à l'Ouest, mais seulement en 1976 à l'Est, et qu'il n'y a plus aucune différence entre les deux groupes côté Ouest durant les 4 dernières années disponibles, alors que les traitements avec phosphore manifestent jusqu'au bout une supériorité côté Est, même si le seuil de signification à 5 p. 100 n'est plus atteint.

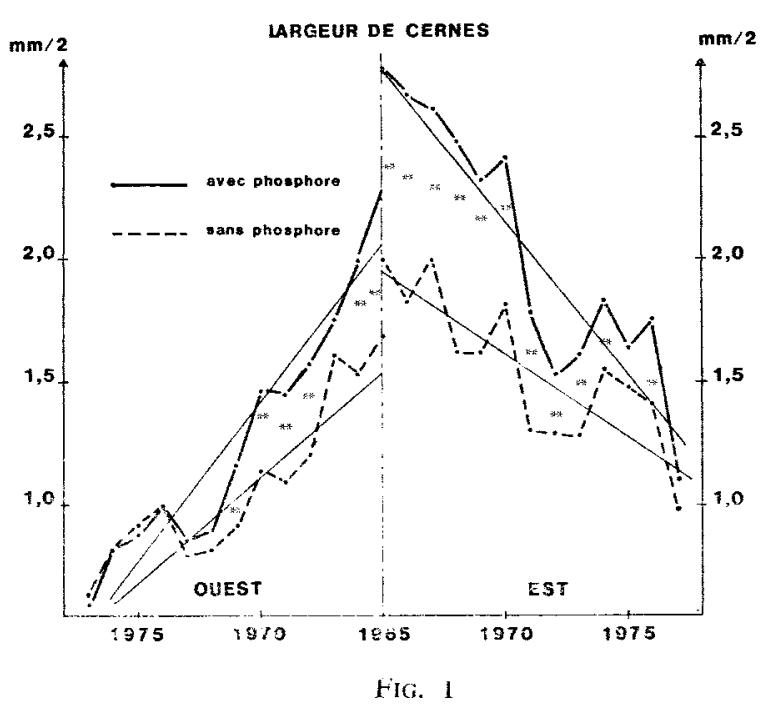

Variations des largeurs de cernes côté bois de compression (Est) et côté opposé (Ouest).

Variations of the ringwidths in the compression wood (East side) and in the opposite wood (West side). 
Sur la figure 2 (partie gauche) est représentée l'évolution dans le temps de l'anisotropie des largeurs de cernes (rapport de l'accroissement sur le plus grand rayon à celui du côté opposé) pour les mêmes deux groupes de traitement; on retrouve, conformément aux observations faites sur les valeurs de $« F »$, une forte augmentation avec l'âge, mais on note aussi, en accord avec les résultats de OHTA et collaborateurs (1983), que cette anisotropie est beaucoup plus marquéc dans les traitements avec phosphore que sans phosphore.
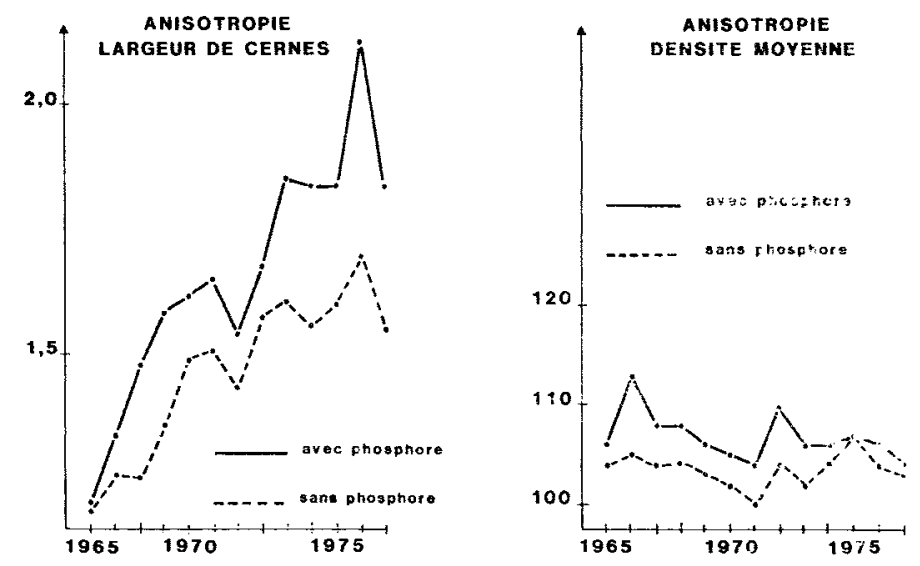

FIG. 2

Variations annuelles de l'anisotropie des largeurs de cernes et des densités movennes.

$V$ ariations of the ringwidth and of the mean density anisotropies.

\subsection{Densités moyennes annuelles}

Le tableau 2 donne pour chaque année la valeur du "F" dû au traitement suivi du test de comparaison des moyennes correspondant :

Les valeurs du test « $F$ » ont tendance à ètre plus faibles que pour les largeurs de cernes, mais il n'apparaît pas d'évolution nette en fonction de l'âge ; less 4 raitements avec phosphore n'ont une densité plus faible que les 3 autres qu'en 1965; par la suite, les 3 dernières places sont occupées, que les différences soient significatives ou non, par un groupement hétéroclite des placeaux $P, N$ et $N P$, cependant qu'alternent à la première le témoin ef le traitement PK.

Si l'on compare ces résultats avec ceux obtenus sur barrettes $7,4 \times 7,4 \mathrm{~mm}$, on note ici des valeurs plus élevées qui s'expliquent par le fait qu'il s'agit maintenant de densités à environ 12 p. 100 d'humidité, et non plus d'infradensité; au reste, les classements se recoupent assez bien dans l'ensemble, et l'on voit, en particulier, que la forte densité du bois des placeaux PK, qui surprenait, s'agissant d'un traitement avec phosphore, est apparue dès 1966 et s'est maintenue avec peu d'exceptions par la suite. 
Tableau 2

Test de comparaison des moyennes des densités moyennes annuelles (valeurs exprimées én $\mathrm{g} / \mathrm{dm}^{3}$ à $12 \mathrm{p} .100$ d'humidité).

Multiple comparisons test of mean annual densities (values given in $\mathrm{g} / \mathrm{dm}^{*}$ at $12 \mathrm{p}$. 100 moisture content).

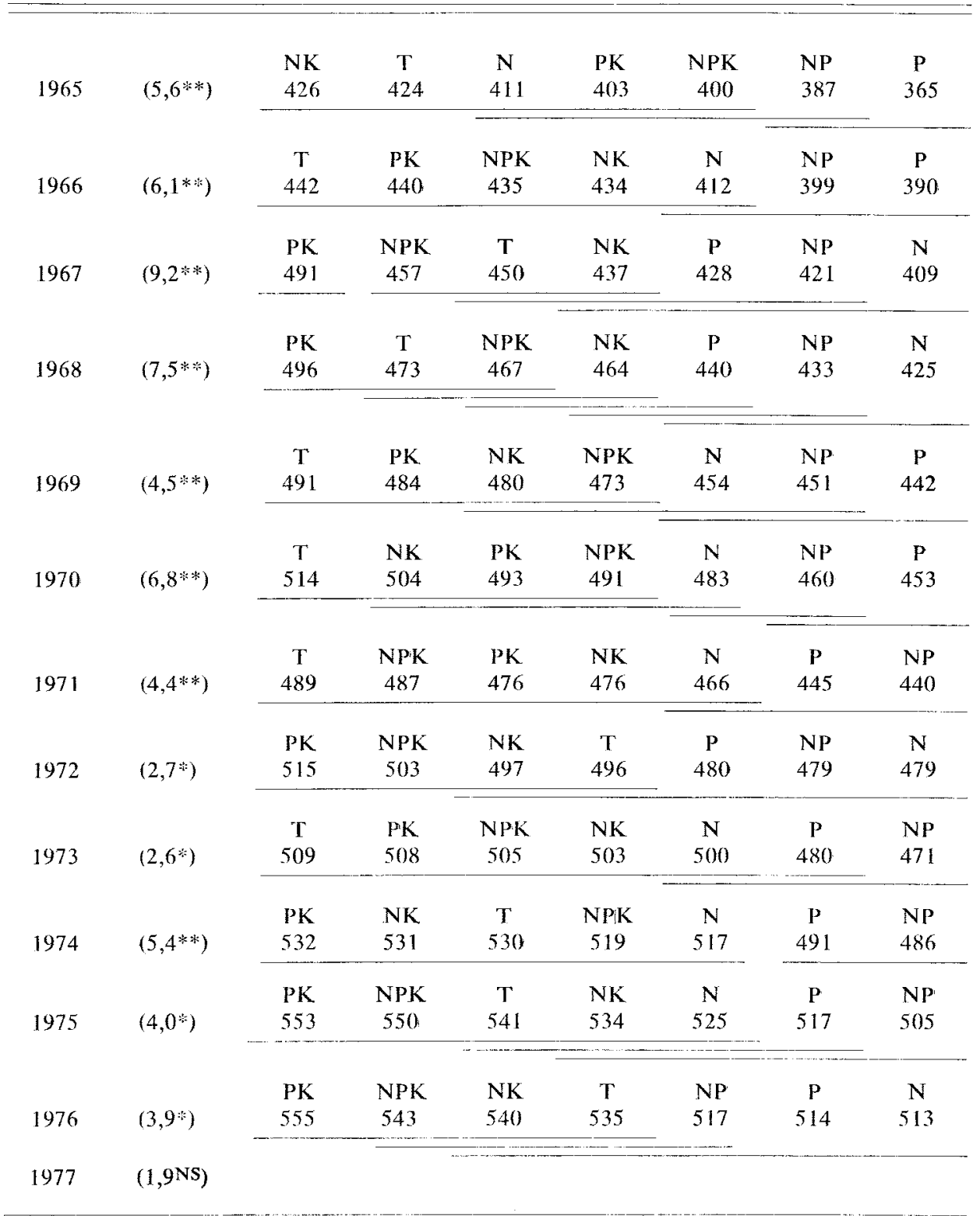


Le test « F » de l'effet côté donne pour les différentes années les valeurs suivantes :

$\begin{array}{ccccccccccccc}1965 & 1966 & 1967 & 1968 & 1969 & 1970 & 1971 & 1972 & 1973 & 1974 & 1975 & 1976 & 1977 \\ 9^{* *} & 35^{* * *} & 15^{* *} & 17^{* * *} & 10^{* * *} & 8^{* *} & 2 \mathrm{NS} & 30^{* *} & 8^{* *} & 16^{* *} & 28^{* * *} & 16^{* *} & 6^{*}\end{array}$

Sans aucune exception, la densité est plus forte du côté bois de compression que du côté opposé.

Il n'y a que 3 années présentant un effet significatif de la taille des arbres, et encore avec des résultats différents : densité plus faible que pour les deux autres catégories pour les petits individus en 1966 et 1967, et pour les moyens en 1974.

Sur la figure 3 sont reportées les valeurs moyennes des densités côté Ouest et côté Est pour la moyenne des traitements avec phosphore d'une part et sans phosphore d'autre part; les différences significatives sont peu nombreuses, ce qui ne saurait surprendre, puisque les tests de comparaison des moyennes ont fait apparaître beaucoup de chevauchements entre les deux groupes; on doit cependant observer que, lorsque le seuil de signification à 5 p. 100 est dépassé, le phosphore entraine toujours une baisse de densité, que les effets de la fertilisation sur la densité du bois sont plus marqués du côté Ouest que du côté Est, que, contrairement aux largeurs de cernes, la dernière différence significative recensée est plus tardive dans le premier (1974) que dans le second (1971), enfin que, d'un côté comme de l'autre, les deux courbes sont maintenant pratiquement confondues, montrant que les effets de la fertilisation sur la qualité du bois, qui s'étaient manifestés dans les expériences précédentes, ont maintenant totalement disparu.

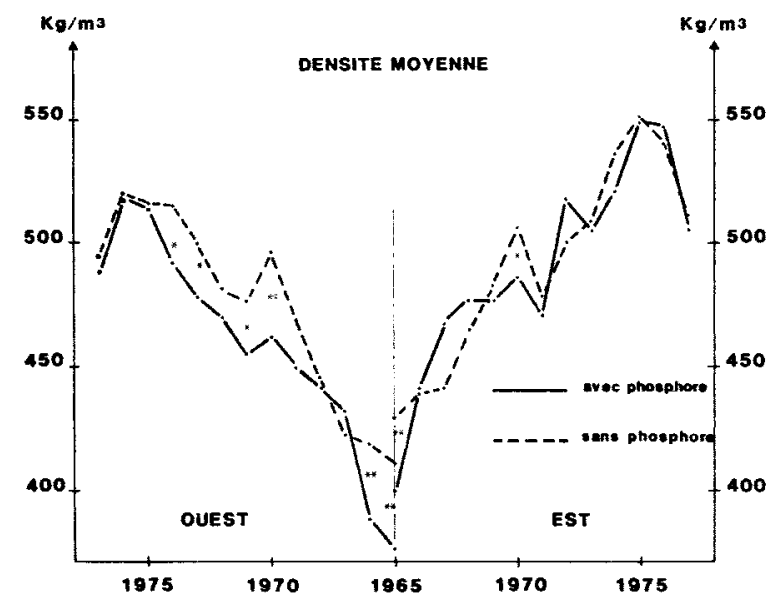

FIG. 3

Variations des densités moyennes annuelles côté bois de compression (Est) et côté opposé (Ouest).

$V$ ariations of the mean annual density in the compression wood (East side) and in the opposite wood (West side). 
Sur la partie droite de la figure 2 , on peut suivre l'évolution dans le temps de l'anisotropie des densités (rapport de la densité Est à la densité Ouest); elle est toujours plus élevée pour les traitements avec phosphore, mais, à l'opposé de ce qui avait été observé pour les largeurs de cernes, celle des placeaux ayant reçu ce type de fertilisation diminue avec l'âge et atteint pour les dernières années des valeurs très voisines de celles des placeaux sans phosphore; ceci est très heureux puisque, d'un point de vue technologique, l'anisotropie des densités est beaucoup plus grave que celle des largeurs de cernes.

\subsection{Densités maximales annuelles}

Les valeurs du test «F $\mathrm{F}$ pour les différentes années et les résultats des comparaisons de moyennes sont donnés dans le tableau 3.

La densité maximale, contrairement à beaucoup d'autres expériences, n'apparaît pas comme particulièrement discriminante pour différencier les traitements, tout au moins dans les 6 dernières années étudiées; c'est la modalité NPK qui, dans l'ensemble, fournit les plus fortes valeurs, cependant que la dernière place est, de façon assez étonnante, occupée tantôt par le témoin, tantôt par le traitement $P$.

La figure 4 résume les variations dans le temps de la densité maximalc pour les deux côtés opposés, et pour les deux groupes de traitement : avec et sans phosphore :

Toutes les différences significatives observées correspondent à une supériorité de la modalité sans phosphore; en outre, comme pour la densité moyenne, le côté Ouest discrimine mieux les deux groupes que le côté Est, mais, contrairement à clle, l'effet dépressif du phosphore est encore apparent en 1977.

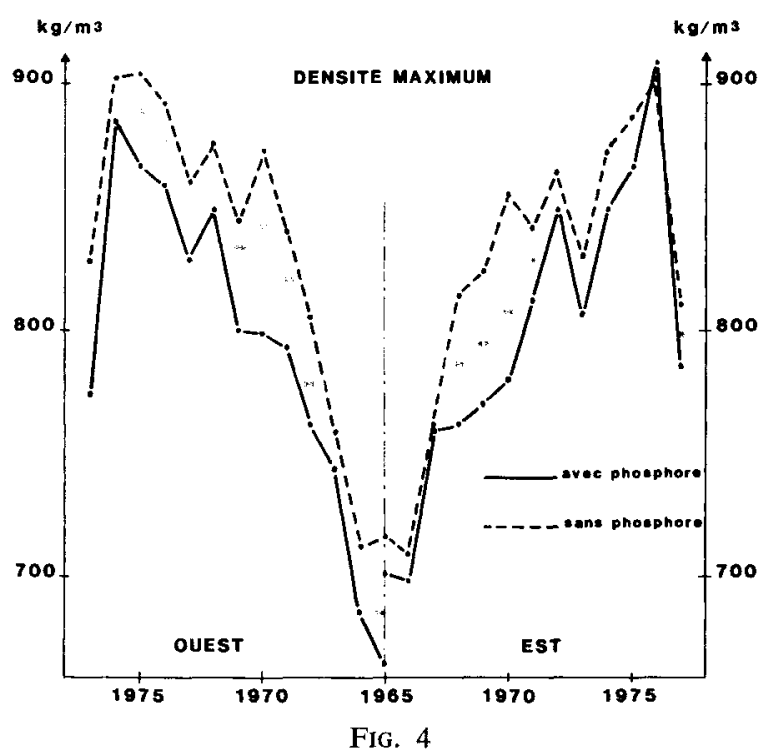

Variations des densités maximales annuelles côté bois de compression (Est) et côté opposé (Ouest).

Variations of the maximum annual density in the compression wood (East side) and in the opposite wood (West side). 


\section{TABLEAU 3}

Test de comparaison des moyennes des densités maximales annuelles (valeurs exprimées en $\mathrm{g} / \mathrm{dm}$ " à 12 p. 100 d'humidité).

Multiple comparisons test of maximum annual densities (in $\mathrm{g} / \mathrm{dm}^{*}$ at $12 \mathrm{p} .100$ moisture content).

\begin{tabular}{|c|c|c|c|c|c|c|c|c|}
\hline 1965 & $(6,3$ ***) & $\begin{array}{l}\text { PK } \\
747\end{array}$ & $\begin{array}{c}\text { NPK } \\
730\end{array}$ & $\begin{array}{c}\mathrm{N} \\
720\end{array}$ & $\begin{array}{l}\text { NK } \\
699\end{array}$ & $\begin{array}{l}\text { NP } \\
673\end{array}$ & $\underset{673}{T}$ & $\begin{array}{c}P \\
646\end{array}$ \\
\hline 1966 & $(5,1 * *)$ & $\begin{array}{c}\text { NPK } \\
747\end{array}$ & $\begin{array}{c}\mathrm{N} \\
730\end{array}$ & $\begin{array}{l}\text { PK } \\
715\end{array}$ & $\begin{array}{l}\text { NK } \\
700 .\end{array}$ & $\begin{array}{c}P \\
675\end{array}$ & $\begin{array}{l}\text { NP } \\
671\end{array}$ & $\begin{array}{c}\mathrm{T} \\
660\end{array}$ \\
\hline 1967 & $\left(5,0^{* * *}\right)$ & $\begin{array}{c}\mathrm{N} \\
794\end{array}$ & $\begin{array}{c}\text { NPK } \\
788\end{array}$ & $\begin{array}{l}\text { PK } \\
770\end{array}$ & $\begin{array}{c}T \\
751\end{array}$ & $\begin{array}{l}\text { NK } \\
748\end{array}$ & $\begin{array}{l}\text { NP } \\
722\end{array}$ & $\begin{array}{c}P \\
712\end{array}$ \\
\hline 1968 & $\left(7,7^{* * *}\right)$ & $\begin{array}{c}\text { NPK } \\
840\end{array}$ & $\begin{array}{l}\text { PK } \\
822\end{array}$ & $\begin{array}{l}\mathrm{N} \\
802\end{array}$ & $\begin{array}{l}\text { NP } \\
770\end{array}$ & $\begin{array}{l}\text { NK } \\
757\end{array}$ & $\begin{array}{c}\mathbf{T} \\
753\end{array}$ & $\begin{array}{c}P \\
739\end{array}$ \\
\hline 1969 & $(7,0 * *)$ & $\begin{array}{l}\mathrm{PK} \\
845\end{array}$ & $\begin{array}{c}\text { NPK } \\
8.38\end{array}$ & $\begin{array}{l}\text { NP } \\
815\end{array}$ & $\begin{array}{c}\mathrm{N} \\
799\end{array}$ & $\begin{array}{l}\text { NK } \\
797\end{array}$ & $\underset{763}{P}$ & $\begin{array}{c}\mathrm{T} \\
749\end{array}$ \\
\hline 1970 & $(14,0 * *)$ & $\begin{array}{c}\text { NPK } \\
882\end{array}$ & $\begin{array}{l}\text { PK } \\
875\end{array}$ & $\begin{array}{l}\text { NP } \\
836\end{array}$ & $\begin{array}{c}\mathrm{N} \\
819\end{array}$ & $\begin{array}{l}\text { NK } \\
806\end{array}$ & $\underset{773}{\mathbf{P}}$ & $\begin{array}{c}\mathbf{T} \\
755\end{array}$ \\
\hline 1971 & $\left(3,7^{* *}\right)$ & $\begin{array}{c}\text { NPK } \\
855\end{array}$ & $\begin{array}{l}\text { NP } \\
8.39\end{array}$ & $\begin{array}{l}\text { PK } \\
836\end{array}$ & $\underset{827}{N}$ & $\begin{array}{l}\text { NK } \\
824\end{array}$ & $\begin{array}{c}\mathrm{T} \\
803\end{array}$ & $\begin{array}{c}P \\
769\end{array}$ \\
\hline $\begin{array}{l}1972 \\
1973\end{array}$ & $\begin{array}{l}\left(1,5^{N S}\right) \\
\left(2,3^{* *}\right)\end{array}$ & $\begin{array}{c}\text { NPK } \\
861\end{array}$ & $\begin{array}{l}\text { NP } \\
846\end{array}$ & $\begin{array}{l}\text { NK } \\
832\end{array}$ & $\underset{831}{N}$ & $\begin{array}{l}\text { PK } \\
830\end{array}$ & $\begin{array}{c}\mathrm{T} \\
819\end{array}$ & $\underset{786}{P}$ \\
\hline 1974 & $\left(2,8^{* *}\right)$ & $\begin{array}{c}\text { PK } \\
890\end{array}$ & $\begin{array}{c}N \\
890\end{array}$ & $\begin{array}{c}\text { NPK } \\
887\end{array}$ & $\begin{array}{l}\text { NP } \\
869\end{array}$ & $\begin{array}{l}\text { NK } \\
869\end{array}$ & $\begin{array}{c}T \\
831\end{array}$ & $\underset{828}{\mathbf{P}}$ \\
\hline 1975 & $(2,7$ * & $\begin{array}{l}\text { PK } \\
9(04\end{array}$ & $\begin{array}{c}\text { NPK } \\
893\end{array}$ & $\underset{892}{N}$ & $\begin{array}{l}\text { NP } \\
891\end{array}$ & $\begin{array}{l}\text { NK } \\
877\end{array}$ & $\begin{array}{c}T \\
863\end{array}$ & $\begin{array}{c}\mathbf{P} \\
836\end{array}$ \\
\hline 1976 & $\left(2,3^{* *}\right)$ & $\underset{933}{N}$ & $\begin{array}{l}\text { NPK } \\
915\end{array}$ & $\begin{array}{l}\text { PK } \\
9 ! 3\end{array}$ & $\begin{array}{l}\text { NK } \\
905\end{array}$ & $\underset{885}{T}$ & $\begin{array}{l}\text { NP } \\
882\end{array}$ & $\underset{86.5}{P}$ \\
\hline 1977 & $\left(2,5^{* * 1 k}\right)$ & $\begin{array}{c}\text { NPK } \\
832\end{array}$ & $\begin{array}{l}\text { PK } \\
827\end{array}$ & $\begin{array}{l}\mathrm{NP} \\
810\end{array}$ & $\begin{array}{c}\mathrm{N} \\
802\end{array}$ & $\begin{array}{c}\mathbf{P} \\
779\end{array}$ & $\begin{array}{l}\text { NK } \\
772\end{array}$ & $\begin{array}{c}\mathbf{T} \\
766\end{array}$ \\
\hline
\end{tabular}




\section{Tablenu 4}

Test de comparaison des moyennes de densités minimales annaelles (valeurs exprimées en $\mathrm{g} / \mathrm{dm}^{2}$ à $12 \mathrm{p}$. 100 d'humidité).

Multiple comparisons test of minimum annual densities (in $\mathrm{g} / \mathrm{dm}^{\mathrm{s}}$ at 12 p. 100 moisture content).

\begin{tabular}{|c|c|c|c|c|c|c|c|c|}
\hline \multirow[t]{2}{*}{1965} & \multirow[t]{2}{*}{$\left(5,6^{* *}\right)$} & $\begin{array}{l}\mathrm{PK} \\
330\end{array}$ & $\begin{array}{c}\text { NP.K } \\
318\end{array}$ & $\begin{array}{l}\text { NP } \\
308\end{array}$ & $\begin{array}{l}\text { NK } \\
293\end{array}$ & $\begin{array}{c}N \\
284\end{array}$ & $\begin{array}{c}P \\
282\end{array}$ & \multirow[t]{2}{*}{$\begin{array}{c}T \\
268\end{array}$} \\
\hline & & & & - & 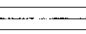 & & - & \\
\hline \multirow{3}{*}{1966} & \multirow{3}{*}{$\left(5,5^{* *}\right)$} & NK & PK & $N$ & NPK & NP & P & ' \\
\hline & & 314 & 314 & 306 & 305 & 293 & 280 & 273 \\
\hline & & $N$ & $\mathrm{PK}$ & NK & NPK & $\mathrm{P}$ & $\mathrm{NP}$ & $T$ \\
\hline 1967 & $(8,2 * *)$ & 338 & 314 & 313 & 311 & 286 & 282 & 280 \\
\hline \multirow[b]{2}{*}{1968} & \multirow[b]{2}{*}{$(7,9 * *)$} & $N$ & NPK & PK & NK & $\mathrm{NP}$ & $P$ & $\mathrm{~T}$ \\
\hline & & 346 & 338 & 330 & 319 & 303 & 296 & 290 \\
\hline \multirow{3}{*}{1969} & \multirow{3}{*}{$\left(7,4^{2}\right)$} & NPK & PK & $\mathrm{N}$ & NK & $N P$ & $\mathrm{P}$ & $\mathrm{T}$ \\
\hline & & 351 & 342 & 330 & 320 & 313 & 304 & 294 \\
\hline & & & & & 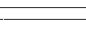 & & & \\
\hline \multirow[b]{2}{*}{1970} & \multirow{2}{*}{$(7,1 * \cdots)$} & $\mathrm{PK}$ & NPK & NK & NP & $N$ & $\mathrm{P}$ & $\mathrm{T}$ \\
\hline & & 353 & 351 & 339 & 333 & 328 & 307 & 299 \\
\hline \multirow{3}{*}{1971} & \multirow{3}{*}{$(5,1 * *)$} & PK & NPK & $N$ & $N P$ & NK & $P$ & $\mathrm{~T}$ \\
\hline & & 354 & 348 & 342 & 338 & 333 & 306 & 30. \\
\hline & & JK & NPK & $N$ & NP & NK & $\mathrm{P}$ & $\Gamma$ \\
\hline 1972 & $(4,7 * *)$ & 345 & 337 & 336 & 328 & .326 & 309 & 305 \\
\hline \multirow[b]{2}{*}{1973} & \multirow[b]{2}{*}{$(5,3 * *)$} & $N$ & PK & $\mathrm{NK}$ & NPK & $\mathrm{NP}$ & $T$ & $P$ \\
\hline & & 371 & 363 & 356 & 355 & 338 & 327 & 323 \\
\hline \multirow[b]{2}{*}{1974} & \multirow[b]{2}{*}{$(4,5 * *)$} & $\mathrm{PK}$ & $N$ & NPK & NK & $N P$ & $P$ & $\mathrm{~T}$ \\
\hline & & 343 & 339 & 339 & 335 & 332 & 308 & 307 \\
\hline \multirow[b]{2}{*}{1975} & \multirow[b]{2}{*}{$(3,9 *)$} & $N$ & PK & NK & NPK & $N P$ & $\mathrm{~T}$ & $\mathbf{P}$ \\
\hline & & 397 & 380 & 379 & 377 & 368 & 355 & 345 \\
\hline \multirow[b]{2}{*}{1976} & \multirow[b]{2}{*}{$(4,4 \div)$} & $\mathrm{N}$ & PK & NK & NPK & NP & $\mathrm{P}$ & $\mathrm{T}$ \\
\hline & & 404 & 388 & 380 & 375 & 369 & 361 & 357 \\
\hline \multirow[b]{2}{*}{1977} & \multirow{2}{*}{$(3,7 * *)$} & $\mathrm{N}$ & NK & NPK & PK & $P$ & $\mathrm{NP}$ & $\mathrm{T}$ \\
\hline & & 375 & 368 & 364 & 355 & 350 & 342 & 340 \\
\hline
\end{tabular}




\subsection{Densités minimales annuelles}

Le tableau 4 donne, par année, la valeur de « $F$ », l'indication des différences significatives entre traitements.

Dans l'ensemble, et surtout les dernières années, la densité minimale paraît plus sensible aux effets de la fertilisation que la densité maximale, mais la hiérarchie des traitements est à peu près la même que pour celle-ci, à la nuance près que l'azote seul semble avoir une influence bénéfique plus marquée. Une remarque s'impose ici : aussi bien pour les minimums que pour les maximums, les valeurs des témoins sont particulièrement faibles, et se différencient même significativement de celles de plusieurs modalités de fertilisation; or, l'inverse était vrai pour les densités moyennes de cernes! Le programme d'analyse densitométrique utilisé ne prévoyait malheureusement pas le calcul des pourcentages de bois final; on en est done réduit aux hypothèses pour cxpliquer ce paradoxe : celle suivant laquelle les lémoins auraient une texture particulièrement forte est la plus vraisemblable, et ce d'autant qu'elle recoupe les résultats obtenus antérieurement sur le pourcentage de l'accroissement annuel ayant une densité supérieure à $500 \mathrm{~g} / \mathrm{dm}^{3}$ (POLge, 1966).

Sur la figure 5 sont indiquées les valeurs annuelles des densités minimales, côté Ouest et côté Est, pour les traitements avec et sans phosphore.

Comme pour les densités maximales, les différences significatives sont toujours au profit des modalités sans phosphore, et la discrimination est meilleure à l'Ouest qu'à l'Est, mais, ici, aucun effet du traitement n'apparaît dans les dernières années de l'expérience.

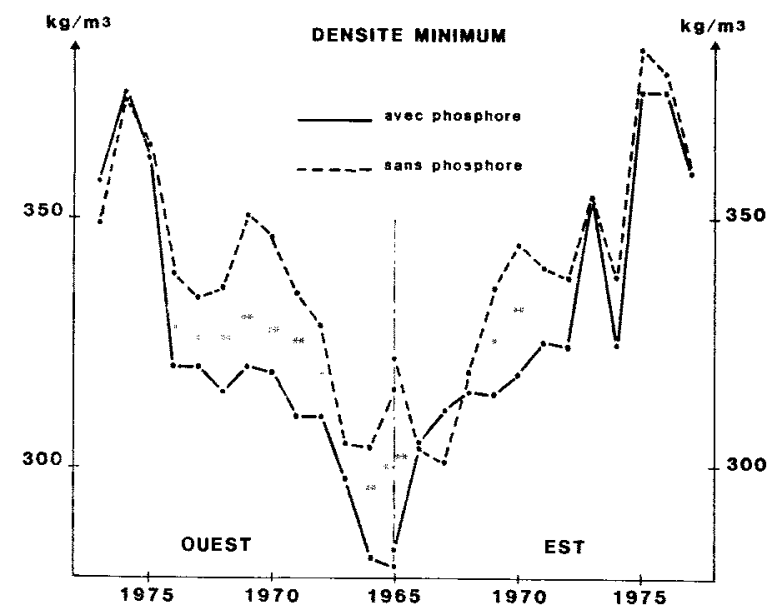

FIG. 5

Variations des densités minimales annuelles côté bois de compression (Est) et côté opposé (Ouest).

Variations of the minimum annual densities in the compression wood (East side) and in the opposite wood (West side). 
3.25. Ecart-type intra-cerne de la densité du bois

Les effets du traitement sont résumés dans le tableau 5.

Ce paramètre, dont on pense qu'il caractérise bien l'homogénéité du bois (Ferrand, 1982), apparaît ici comme peu apte à différencier les traitements ; seules, 4 années donnent un « $F$ » significatif, et la hiérarchie des types de fertilisation varie fortement de l'une à l'autre, mis à part les placeaux NPK à forts écarts-types, et les placeaux $\mathrm{P}$ beaucoup plus homogènes.

\section{TABLEAU 5}

Test de comparaison des moyennes de l'écart-type des densités (valeurs exprimées en $\mathrm{g} / \mathrm{dm^{3 }}$ ).

Multiple comparisons test of within ring densities standard error (in $\mathrm{g} / \mathrm{dm} \mathrm{m}^{3}$ ).

\begin{tabular}{|c|c|c|c|c|c|c|c|c|}
\hline 1965 & $(1,9 N S)$ & & & & & & & \\
\hline 1966 & $(2,0 \mathrm{NS})$ & & & & & & & \\
\hline 1967 & $(1,7 \mathrm{NS})$ & & & & & & & \\
\hline & & NPK & PK & $T$ & $N$ & $\mathrm{NP}$ & NK & $P$ \\
\hline 1968 & $\left(2,8^{k}\right)$ & 150 & 148 & 139 & 137 & 133 & 132 & 131 \\
\hline 1969 & $(1,1 \mathrm{NS})$ & & & & & & & \\
\hline \multirow{3}{*}{1970} & & NPK & PK & NP & $\mathrm{N}$ & NK & $P$ & $\mathrm{~T}$ \\
\hline & $(3,9 * *)$ & 173 & 169 & 159 & 157 & 154 & 149 & 147 \\
\hline & & $T$ & NPK & NK & NP & $\mathbf{N}$ & PK & $\mathrm{P}$ \\
\hline 197 I & $(2,9 *)$ & 157 & 154 & 152 & 151 & 143 & 141 & 136 \\
\hline 1972 & $(1,4 \mathrm{NS})$ & & & & & & & \\
\hline 1973 & $\left(2,0^{N S}\right)$ & & & & & & & \\
\hline 1974 & $(1,7 \mathrm{NS})$ & & & & & & & \\
\hline 1975 & $(0,9 \mathrm{NS})$ & & & & & & & \\
\hline 1976 & $\left(1,6^{\mathrm{NS}}\right)$ & & & & & & & \\
\hline \multirow{2}{*}{1977} & $\left(3,6^{* *}\right)$ & PK & NPK & NP & $T$ & $\mathrm{~N}$ & $P$ & NK \\
\hline & & 159 & 158 & 155 & 146 & 142 & 140 & 132 \\
\hline
\end{tabular}

3.26. Effets secondaires sur les densités maximales et minimales et sur les écarts-lypes

Ils sont regroupés ici, car ils sont peu marqués et sans grande implication pratique; seules seront indiquées les années à différences significatives.

- Effet du côté :

Contrairement aux largeurs de cernes et aux densités moyennes, très peu d'années sont concernées; elles figurent sur le tableau 6. 


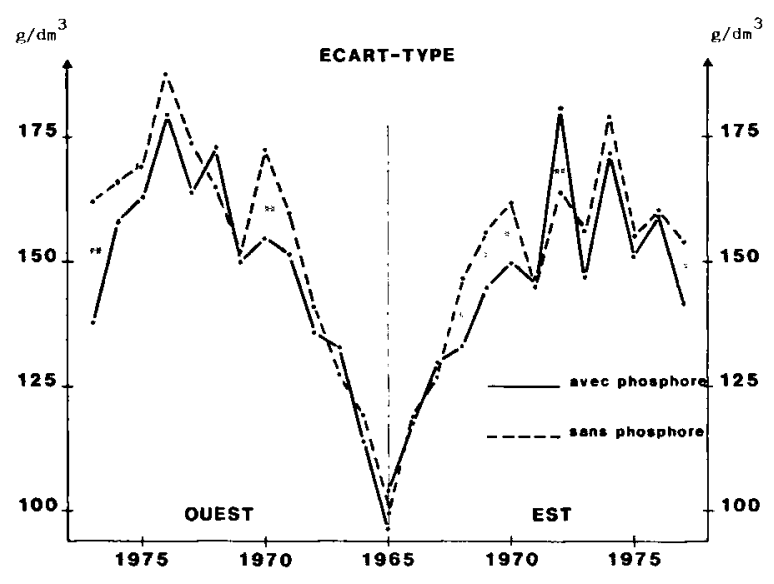

FIG. 6

Variations de l'écart-type des densités.

Variations of the standard error of densities.

Avec phosphore - With phosphorus.

Sans phosphore - Without phosphorus.

\section{TABLeau 6}

Analyse de l'effet "coité sur les critères amnuels de densité.

Analysis of the side effect on the densitometric criteria.

\begin{tabular}{l|c|c|c|c|c}
\hline & Annéc & F & Côté Est & Côté Ouest & \\
\hline & & & & & \\
\hline & 1966 & $6,6^{*}$ & 304 & 291 & $\mathrm{~g} / \mathrm{dm}^{3}$ \\
& 1973 & $4,9^{*}$ & 354 & 341 & \\
Densités minimales annuelles & 1975 & $5,6^{*}$ & 379 & 363 & \\
& 1973 & $5,0^{*}$ & 816 & 842 & $\mathrm{~g} / \mathrm{dm}^{3}$ \\
& 1970 & $4,3^{*}$ & 155 & 162 & $\mathrm{~g} / \mathrm{dm}^{3}$ \\
& 1973 & $14,7^{* *}$ & 151 & 168 & \\
\hline
\end{tabular}

Année - year ; côté - side; Est - East; Ouest - West; densité - density ; annuel - annual; écart-type - stamdard error.

Les densités maximales sont plus faibles, et les minimales plus élevées du côté comprimé que du côté opposé ; corrélativement, le bois y est plus homogène.

- Effet de la grosseur :

Il se manifeste très peu sur les maximums et sur les minimums, mais davantage sur les écarts-types; les résultats sont les suivants : 


\section{Tableau 7}

Effets de la grosseur des arbres sur les critères annuels de densité.

Effects of the tree size on the densitometric criteria.

\begin{tabular}{|c|c|c|c|c|c|c|}
\hline & Annéc & $\mathrm{F}$ & & & & \\
\hline \multirow[t]{2}{*}{ Densités minimales annuelles } & 1973 & $4,6 *$ & M : 334 & $G: 354$ & $P: 355$ & \multirow[t]{2}{*}{$\mathrm{g} / \mathrm{dm}^{3}$} \\
\hline & 1977 & $5,7 *$ & M : 344 & $G: 360$ & $P: 365$ & \\
\hline \multirow[t]{2}{*}{ Densités maximales annuclles } & 1970 & $5,4 * * *$ & $\mathrm{G}: 804$ & $M: 815$ & $P: 842$ & \multirow[t]{2}{*}{$\mathrm{g} / \mathrm{dm}^{3}$} \\
\hline & 1974 & $4,7:$ & M : 848 & $\mathrm{G}: 857$ & $P: 892$ & \\
\hline \multirow[t]{6}{*}{ Ecarts-types des densités } & 1965 & $4.4^{*}$ & $P: 94$ & $\mathrm{M}: 103$ & $G: 103$ & \multirow[t]{6}{*}{$\mathrm{g} / \mathrm{dm}^{3}$} \\
\hline & 1967 & $3,3: *$ & $\mathrm{G}: 123$ & $P: 129$ & M: 135 & \\
\hline & 1970 & $4,4 *$ & $\mathrm{G}: 152$ & M : 158 & $P: 165$ & \\
\hline & 1974 & $3.5 *$ & $G: 174$ & M : 177 & $P: 187$ & \\
\hline & 1975 & $4.0^{*}$ & $\mathrm{G}: 155$ & $M: 156$ & $P: 167$ & \\
\hline & 1977 & $3,4 *$ & $\mathrm{G}: 141$ & M : 147 & $P: 154$ & \\
\hline $\begin{array}{l}\mathbf{P}=\text { Petits arbres - small } \\
\mathbf{M}=\text { Arbres moyens - mean } \\
\mathrm{G}=\text { Gros arbres - large we }\end{array}$ & trees. & & & & & \\
\hline
\end{tabular}

Pout les densités maximale et minimale annuelles, on n'a que deux années où la taille des arbres apparaît comme un facteur discriminant, mais, dans le premier cas, c'est la calégorie des petits arbres qui se différencie des deux autres par une valeur élevée, alors que, dans le second, e’est la catégorie moyenne, par dés valeurss faibles.

La situation fluctue quelque peu pour l'écart-type des densités, mais la tendance générale est celle d'une amélioration de l'homogénéité en passant des petits aux moyens, puis aux gros.

\subsection{Essais sur éprouvettes normalisées}

\subsection{Fleche à la rapture en flexion statique}

Le résultat des comparaisons de moyennes au seuil de 5 p. 100 (valeurs données en $\mathrm{mm}$ ) et la valeur du test « $F$ » sont donnés ci-dessous :

\begin{tabular}{ccccccr} 
NP & P & PK & N & NK & T & NPK \\
10,4 & 10,9 & 10,9 & 11,0 & 11,3 & 11,4 & $12,0 \quad \mathrm{~F}=2,3$ \\
\hline
\end{tabular}


L'éventail des moyennes par traitement est plus refermé que dans l'étude de OHтA et al., 1983 ; on retrouve une tendance générale à un effet négatif du phosphore sur la flèche à la rupture, mais, alors que dans cette expérience précédente la principale anomalie était constituée par la présence insolite des témoins parmi les traitements à phosphore, ici, on est surpris par la forte valeur trouvéc pour le traitement NPK.

\subsection{Contrainte de rupture en flexion statique}

Les variations entre traitements se présentent comme suit (valeurs en $\mathrm{daN} / \mathrm{cm}^{2}$ ) :

\begin{tabular}{cccccccc} 
NP & PK & NPK & P & N & NK & T \\
629 & 691 & 709 & 716 & 745 & 764 & 815 & $F=12,4 \%$ \\
\hline
\end{tabular}

Pour cette caractéristique également, les chiffres trouvés sont nettement moins dispersés que ceux obtenus sur du matériel prélevé 6 ans plus tôt ; mais on trouve surtout un meilleur regroupement des traitements avec et sans phosphore, avec, en particulier une séparation complète de $\mathrm{T}$ et $\mathrm{NK}$ d'une part, et de l'ensemble des modalités de fertilisation avec $P$, seul ou combiné, d'autre part.

\subsection{Dureté}

Les résultats sont les suivants (en $\mathrm{mm}$ de largeur d'empreinte) :

\begin{tabular}{cccccccc}
$\mathrm{T}$ & $\mathrm{N}$ & $\mathrm{PK}$ & $\mathrm{NK}$ & $\mathrm{NPK}$ & $\mathrm{P}$ & $\mathrm{NP}$ \\
7,8 & 8,2 & 8,4 & 8,4 & 8,9 & 9,0 & $10,7 \quad \mathrm{~F}=13,9^{* * *}$ \\
\hline
\end{tabular}

Les valeurs indiquées ne sont pas comparables à celles données par OHTA et collaborateurs qui avaient préféré chiffrer la dureté en profondeur d'empreinte plutôt qu'en largeur (les deux sont liées par une relation simple faisant intervenir le rayon du cylindre d'appui); la dispersion est, à nouveau, un peu plus faible ici, mais la valeur du test « $\mathrm{F}$ » plus élevée; ici, trois des 4 traitements avec phosphore se différencient significativement des trois modalités sans phosphore, avec des valeurs supérieures traduisant une moindre dureté.

\subsection{Contrainte de rupture en compression axiale}

La comparaison des traitements donne (en $\mathrm{daN} / \mathrm{cm}^{2}$ ) :

$\begin{array}{ccccccc}\text { NP } & \text { NPK } & \text { NK } & \text { P } & \text { T } & \text { PK } & \text { N } \\ 402 & 442 & 445 & 451 & 458 & 462 & 479\end{array}$

Les valeurs trouvées sont toujours moins dispersées que dans l'étude précédente (ce qui pourrait s'expliquer par le fait qu'il est de moins en moins difficile de confectionner des éprouvettes de bonne qualité en nombre suffisant au fur et à mesure que les arbres grossissent), mais, ici, on trouve en plus des chiffres de contrainte supérieurs, ce qui pourrait résulter d'une sensibilité plus grande de l'essai en compression axiale à la présence de petits défauts dans les éprouvettes; on doit cependant noter que la séparation des traitements avec et sans phosphore est maintenant moins bonne que 6 ans plus tôt. 


\section{Commentaires et conclusion}

Cette nouvelle série d'expériences confirme un certain nombre de résultats antérieurs, et apporte quelques éléments d'information nouveaux :

Il est tout d'abord clair que l'influence défavorable de la fertilisation phosphatée sur la densité du bois et, par voie de conséquence, sur sa résistance, qui n'a jamais présenté un caractère dramatique, s'estompe de plus en plus, ct est sans doute maintenant en voie de disparition; si on l'a encore observée ici sur plusieurs critères de comportement mécanique, la raison en est que, dans les éprouvettes d'essai, étaient obligatoirement représentés un certain nombre de cernes anciens sur lesquels l'effet du traitement était toujours sensible.

On est certes surpris par des anomalies apparentes de classement observées dans les tests de résistance en flexion, compression axiale ou dureté, et aussi par le fait qu'elles sont différentes de celles notées par OHTA et ses co-auteurs en 1983 ; ceci est vraisemblablement dô à l'action du climat sur la structure du bois, donc sur son comportement rhéologique, qui vient interférer avec celle de la fertilisation dans les phénomènes de nutrition: il faut se rappeler en effet que les différences de densité entre années, sous l'effet des variations des divers facteurs climatiques, sont telles qu'elles sont à l'origine de la xylochronologie, c'est-à-dire de la datation du bois basée sur les seules données densitométriques (Polgk, 1966; Polge \& Keller, 1969); les éprouvettes des deux séries d'essais ayant obligatoirement été constituées de cernes différents, l'interaction climat-fertilisation peut très bien ne pas s'être exercée de façon identique, et avoir entraîné, sur l'anatomie du bois formé, des effets, sinon opposés, du moins dissemblables.

Cette expérience a confirmé tout l'intérêt qu'il y a, pour le pin maritime des Landes, à étudier séparément le bois du côté Est (bois de compression) et le bois opposé : pour la plupart des caractéristiques analysées ici : retraits tangentiel, radial et axial, largeurs de cernes, densités moyennes annuelles, écarts-types des densités, les valeurs du test « $F$ » de l'effet côté sont supérieures à celles de l'effet traitement; l'influence du phosphore comme facteur d'aggravation de l'anisotropie des largeurs de cernes, déjà observée lors de l'expérience précédente, s'est trouvée confirmée dans celle-ci, mais on a surtout pu noter que, fort heureusement, si cette anisotropie augmente avec l'âge, notamment dans les traitements avec phosphore, celle des densités évolue en sens contraire.

L'effet de la fertilisation sur les dimensions axiales et tangentielles des éprouvettes débitées avec des seies-fraises jumelées pourrait paraitre ne présenter qu'un intérêt anecdotique; cela n'est pas sûr : certes les différences entre traitements extrêmes, bien que significatives, ne représentaient pas 1 p. 100 en valeur relative, ct pouvaient donc être considérées comme négligeables pour l'analyse densitométrique ; il pourrait ne pas en être de même avec d'autres sources de variations plus efficaces (génétique, sylviculture, élagage) dont on sait qu'elles conditionnent puissamment la densité du bois, et il conviendrait sans doute de porter d'autant plus attention à l'uniformité d'épaisseur des échantillons après débit que sur elle repose, non seulement la détermination des composantes de la densité par absorption des rayons $X$, mais aussi celle des modules d'élasticité par atténuation de la vitesse des ondes ultrasonores (Voïchita Bucur, 1981 ; J.R. Perrin, 1983). 
Si de nouveaux efforts de recherches devaient être consacrés aux effets de la fertilisation sur la qualité du bois de pin maritime, et cela serait sans doute justifié par l'importance des forêts ainsi traitées dans la région landaise, il pourrait être utile de revenir à des prélèvements non-destructifs de façon à pouvoir analyser, d'une part l'éventuel effet positif du potassium sur la densité du bois, d'autre part l'interaction entre facteurs du climat et éléments fertilisants, qui n'ont pas pu être tirés au clair dans cette expérience faute d'un échantillonnage suffisant.

Regut en août 1984.

Accepté en novembre 1984.

\section{Remerciements}

Les Papetcries de Gascogne à Mimizan ont fourni gracieusement les billons nécessaires à cette expérience,

Bernard Lemolne s'est assuré de la représentativité statistique de l'échantillonnage,

Danièle Aubert a procédé à la totalité du traitement des donnécs;

Que tous soient ici remerciés de l'aide précieuse ainsi apportée à cette étude.

\section{Summary}

\section{Further study of the effects of fertilization on wood quality of maritime Pine (Mimizan experiment)}

From each of 105 trees distributed between 5 blocks, 7 fertilization treatments and 3 diameter classes, 2 small strips $7.4 \times 7.4 \mathrm{~mm}$ were cut, one along a radius on the side of the compression wood (East), the other on the opposite side, each divided into samples near the pith and near the bark; observations of basic density showed no clear separation between treatments with and without phosphorus, the smaller trees having the less dense wood and the eastern side denser wood; there wore significant differences between treatments of the thickness of specimens cut with twin circular saws; radial and tangential shrinkages were lower near the pith than near the bark, and lower in the eastern than in the western direction whercas the contrary was observed for longitudinal shrinkage; fertilization influenced the shrinkages, but it is difficult to show an effect of individual chemical elements.

Standardized specimens showed a rather good separation between treatments, particularly as regards breaking strength in static bending and hardness which were lower in the case of treatments with $P$.

A densitometric analysis performed on $1.6 \mathrm{~mm}$ thick specimens showed that the beneficial influence of $P$ on ring width ceases earlier in the compression wood than in the normal wood; the effect of the tree size increased with age; the anisotropy of the ring widths was higher for the treatments with $P$, alone or combined with other elements; there were significant differences between treatments for the mean, the maximum and the minimum annual densities, but without any clear effects corresponding to the three elements $(\mathrm{P}, \mathrm{N}$ and $\mathrm{K})$; however, for some individual years, the treatments with and without $P$. respectively, produced significant differences, the values being always lower when $P$ had been used. The anisotropy of ring width increased with age, and that of the mean annual density decreased.

On the whole, the influence of fertilization on wood quality seems never very strong and practically disappears in the last few rings in 21 years old trees. 


\section{Références bibliographiques}

Bucur Voïchita, 1981. Détermination du module d'Young du bois par une méthode dynamique sur carottes de sondage. Ann. Sci. For., 38 (2), 283-298.

FERrand J.Ch., 1981. Recherches des solutions pratiques à apporter aux problèmes posés par les contraintes de croissance des arbres forestiers. Thèse de Docteur-Ingénieur en Sciences du Bois, I.N.P.L. Nancy.

Ferrand J.Ch., 1982. Réflexions sur la densité du bois. 2" partie : Calcul de la densité et de son hétérogénéité. Holzforschung, 36, Heft 3, 153-157.

Gelpe J., Guinaudeau J., 1974. Essai de fertilisation minérale sur Pins maritimes à Mimizan (Landes). Résultats après la $16^{\circ}$ année. Rev. for. fr., 6, 459-463.

Guinaudeau J., llly G., Mauge J.P., Dumas F., 1963. Essai de fertilisation minérale sur pin maritime à Mimizan (Landes). Ann. Sci. For., 20 (1), 1-71.

Ohta S., Keller R., Janin G., 1983. Essai de divers modes de fertilisation sur certaines caractéristiques physiques, chimiques, mécaniques et propriétés papetières du pin maritime des Landes (Pinus pinaster). I. - Caractéristiques physiques, chimiques et mécaniques. Ann. Sci. For., 40 (3), 283-298.

Ohta S., Kel.t.r R., Janin G., 1985. Effets de divers modes de fertilisation (N, P, K) sur certaines caractéristiques physiques, chimiques, mécaniques et propriétés papetières du pin maritime des Landes (Pinus pinaster Ait.). II. - Bois de compression et propriétés papetières. Ann. Sci. For., 42 (1), 69-95.

Perrin J.R., 1983. Dispositif de sciages de carottes de sondage de $5 \mathrm{~mm}$ sans collage préalable sur un support. Ann. Sci. For., 40 (4), 399-406.

Polge H., 1966. Etablissement des courbes de variation de la densité du bois par exploration densitométrique de radiographie d'échantillons prélevés à la tarière sur les arbres vivants - Applications dans les domaines technologique et physiologique. Thèse de Docteur ès Sciences Appliquées, Faculté des Sciences de l'Université de Nancy.

Polge H., llly G., 1967. Observations sur l'anisotropie du pin maritime des Landes. Ann. Sci. For., 24 (3), 205-231.

Polge H., 1969. Influence de la fertilisation sur la qualité du bois de pin maritime. Ann. Sci. For., 26 (1), 45-64.

Polge H., Keller R., 1969. La xylochronologie, perfectionnement logique de la dendrochronologie. Ann. Sci. For., 26 (2), 225-256.

Polge H., 1978. Fifteen years of wood radiation densitometry. Wood Science and Technology, 12, 187-196. 